\title{
Development and Research of Digital Campus System Based on Android
}

\author{
$\mathrm{Li} \mathrm{Ma}^{1,2,3}$, Yu Zhou ${ }^{1,2}, \mathrm{KuoLiao}^{3,4}$, Shen Liu ${ }^{1,2}$, Jie Qiao ${ }^{1,2}$, \\ Zhenyu Han ${ }^{1,2}$ and Jin Wang ${ }^{1,2}$ \\ ${ }^{1}$ Nanjing University of Information Science and Technology, Network Monitoring \\ Center of Jiangsu Province, Nanjing 210044 \\ ${ }^{2}$ Nanjing University of Information Science and Technology, College of Computer \\ and Software, Nanjing 210044 \\ ${ }^{3}$ Nanjing University of Information Science and Technology, Key Laboratory of \\ Meteorological Disasters of Ministry of Education, Nanjing 210044 \\ ${ }^{4}$ Ningde Meteorological Bureau, Ningde 352100 \\ Abstract
}

\begin{abstract}
Digital Campus System is an important platform for student to get all kinds of information. Since the present digital campus system has a defect that wsers can't achieve the campus information through a mobile phone this paper focuse on some key issues such as how to connect to the target site via the communication interface, crawl pages and sub-pages, parse the desired content and store information in the SQlite database in developing wireless digital campus system based on Android. As a result, this paper provides a practical solution to access School Dean, libraries, etc. Compared with the traditional way to access Web pages through the mobile browser, the software is more convenient and saves much more data traffic. At the same time, the software not only achieves the docking of Mobile and campus information, and bring a great convenience to the majority of students, but also providesefficient support for the school management.
\end{abstract}

Keywords: Wireless Digital Campus, HTTP client, Cookie, Jsoup resolution, Android

\section{Introduction}

School students' deman for smart IT is the most exuberant, and its group identity decides that it can be a Frontier which helps promote and develop information technology. Traditional digital eampus system is a campus server which is based on Computer network and makng PC as a terminal device to achieve all information resources, relating to the school, teaching, research, management, and living service, is fully digitized and stored, expanding the real campus to the off campus. However, traditional digital campus system can't realize the information exchange and information services at any time or anywhere. Moreover, these information services are based on the PC. Nowadays, with the development of wireless network technology, mobile communication technology (especially $3 \mathrm{G}$ technology) and the further development of university education reform, many universities construct multiple wireless access points on campus and make the use of mobile phones or other mobile devices to access the network more increasingly widespread. And since mobile communication technology is time efficiency and convenient, it's meaningful to deeply dig the applications which applies the mobile communication technology to help student exchange and communicate. A real market demand for exploring the university information technology construction and building a campus information exchange platform which is based on mobile communication technology can be told.

With the rapid proliferation of smart phones and the rapid development of its related 
technologies, smart phones occupy a high market share in a number of handheld devices. And smart phones regarding the Android as operating system account for a large proportion of all the smart phones. The campus information is enormous, it's normal for today's college students to view class locations and test results, renew books from the library, browse all kinds of campus information. There're some mobile applications about helping students to achieve campus information, but the vast majority of them are only developed with a particular function. Thus, it's only convenient for students to conduct a unilateral operation instead of bringing a variety of convenience for students in the true sense. Not only does the software achieve the docking of Mobile and campus information and bring a great convenience to the majority of students, but also provide effective support for the school management.

\section{Solutions for Communication Interface}

Two ways to develop website clients have been developed. The first is calling the open platform server and calling the API interface to develop. The second is that the server does not provide any interface or any access to any database, developers yrite the communication interface of websites on their own. The system uses the second method to develop and accesses data indirectly via the communication interface. As long as the content can be seen on the website, the client can gain the information The process of accessing data is shown in Figure 1.

\subsection{Methods}

\section{Figure 1. The Process of Obtaining Information}

In the development of Android system, two main methods to access the network for HTTP are developed. The first one is using HttpURL-Connection. The second one is using HttpClient. Generaly, if developers only need to submit a request to a simple page and get response from the server, he can use the first method. But for more complex network operations, using HttpURLConnection class may not be able to meet the requirements. In the situation like this, developers can apply the second method. In this paper, the program uses the second method to achieve the communication interface. It has been successfully to integrate HttpClient in Android, so developers can use Http-Client to access the network directly in Android. HttpClient sends HTTP requests in two ways, GET requests and POST requests. Normally, GET method focuses on requesting web with simple data, POST method focuses on requesting web with complex data. In this paper, the program all uses POST method to send requests. The processes of requesting POST are shown as follows:

(1)Create a HttpClient object,

(2)Create a HttpPost object,

(3)If the program needs to send a request parameter,connect directly the request parameter to the URL address. Or call the setParams () method of HttpPost to add the request parameters.

(4)Call excute () method of HttpClient object to send the request. Implementation of the method is to return an HttpResponse object. 
(5) Call getEntity () method of HttpResponse, developers can get the HttpEntity object containing the responsing content of the server, and the responsing content of the server can be obtained by the object.

\subsection{Program Implementation}

Two steps are made to achieve the communication interface. First, use HttpWatch tools to get POST Data of the site. Second, pass the parameters which are needed to the target site. Detailed steps are as follows:

(1) Use HttpWatch tools to get POST Data of the site. Take the parameters of searching th e website of library for example. Figure 2 shows four parameters required for the site. As t he figure shows, they are "number", "passwd", "retrunUrl" and "select", in which, "numb er" represents student ID, "passwd" represents password, "retrunUrl" represents a null val ue, and "select" represents the type of account login. These four parameters are the paran eters which POST request method requires.

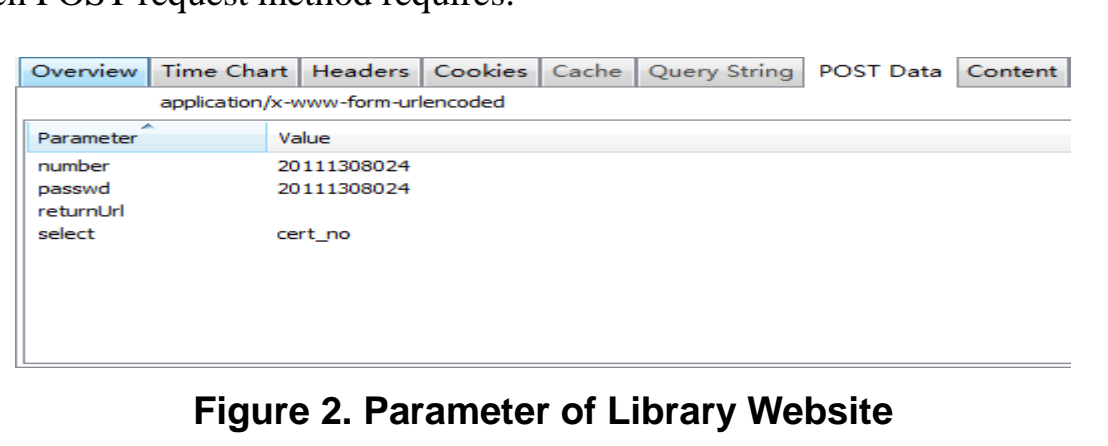

(2) Pass the required parameters to the target site, then access returned data from the site. Take the parameters of searching the website of library for example, the key code of this s tep are as follows:

List<Cookie> cookies
HttpClient client = new DefaultHttpdlient();
HttpResponse httpResponse;

List $<$ NameValue Rair $>$ params $=$ new ArrayList $<$ NameValuePair $>()$;

HttpPost httpRequest = new HttpPost(url);

params.add(new BasicNameValuePair("number",number));

params.add(new Basie1)ameValuePair("passwd",passwd));

params.add(new BasicNameValuePair("returnUrl",""));

params.add(new BasicNameValuePair("select","cert_no"));

try \{

httpRequest.setEntity(new UrlEncodedFormEntity(params,HTTP.UTF_8));

httpResponse $=$ client.execute(httpRequest);

if (httpResponse.getStatusLine().getStatusCode $($ ) == HttpStatus.SC_OK)

StringBuffer $\mathrm{sb}=$ new StringBuffer();

HttpEntity entity $=$ httpResponse.getEntity () ;

InputStream is = entity.getContent();

BufferedReader br = new BufferedReader(new InputStreamReader(is, "utf_8"));

String data $=$ "";

while $(($ data $=$ br.readLine ()$) !=$ null $)\{$

sb.append(data);

\}

Html_doc=sb.toString(); 
\} else \{

//Connection Failed

\}

\} catch (Exception e) \{

e.printStackTrace();

\}

\section{Principles and Methods of Web Crawling}

Typically, Using HttpClient way to visit the website needed to submit parameters, the content users get is the content after the website's jump. Data on this page is often unnecessary, instead, the content of the other sub-sites through this website jumping is the necessary one. Web crawling process is shown in Figure 3.

\subsection{Methods}

Figure 3. Web Crawling Process

In this paper, the program accesses other sub-pages of the website landing page by carrying Cookie. Cookie is commohly known as "cookies", which solves the HTTP protocol itself stateless. It is developed by Netscape (Netscape) as an effective tool for saving user identification information. Essentrally, Cookie can be seen as an license, that means, in the following pages operations, Cookie can prove that its object is the current user instead of others. The specific methods of Web crawling are shown as follows:

(1)Create a HttpClient object;

(2)Create a HttpPost object?

(3)Call setParams () method of HttpPost to add the request parameters;

(4)Call excute () metnod of HttpClient object to send the request. Implementation of the method will returnan HttpResponse object;

(5)Get returns of Cookie;

(6)Create a second HttpPost object;

(7)Put Cookie into the header information;

(8)If the program needs to send a request parameters, call setParams () method of Http os to add the request parameters;

(9)Call excute () method of HttpClient object to send the request. Implementation of the method will return an HttpResponse object;

(10)Call getEntity () method of HttpResponse to obtain HttpEntity object which contains t he content of server response, the user can get the content of response server through the $o$ bject.

\subsection{Program Implementation}

Put the Cookie header into the message, send a POST request to the target site, then, the program can access the content of site response called the site HTML document. Take visiting the library's "Books to borrow" this sub-pages as an example, Figure 4 shows the 
site information, Figure 5 shows the effect after client resolver. The key code is as follows:

cookie $=$ cookies.get $(0)$.getValue () ;

HttpPost httpRequest = new HttpPost(url);

try \{

httpRequest.setHeader("Cookie","PHPSESSID="+ cookie);

HttpResponse httpResponse = new DefaultHttpClient().execute(httpRequest);

if (httpResponse.getStatusLine().getStatusCode ()$==$ HttpStatus.SC_OK) \{

StringBuffer $s b=$ new StringBuffer();

HttpEntity entity $=$ httpResponse.getEntity();

InputStream is = entity.getContent();

BufferedReader br = new BufferedReader(new InputStreamReader(is, "utf_8"));

String data = "";

while $(($ data $=$ br.readLine ()$) !=$ null $)\{$

sb.append(data);

\}

Html_doc=sb.toString () ;

\}

\} catch(Exception e) \{

e.printStackTrace(); \}

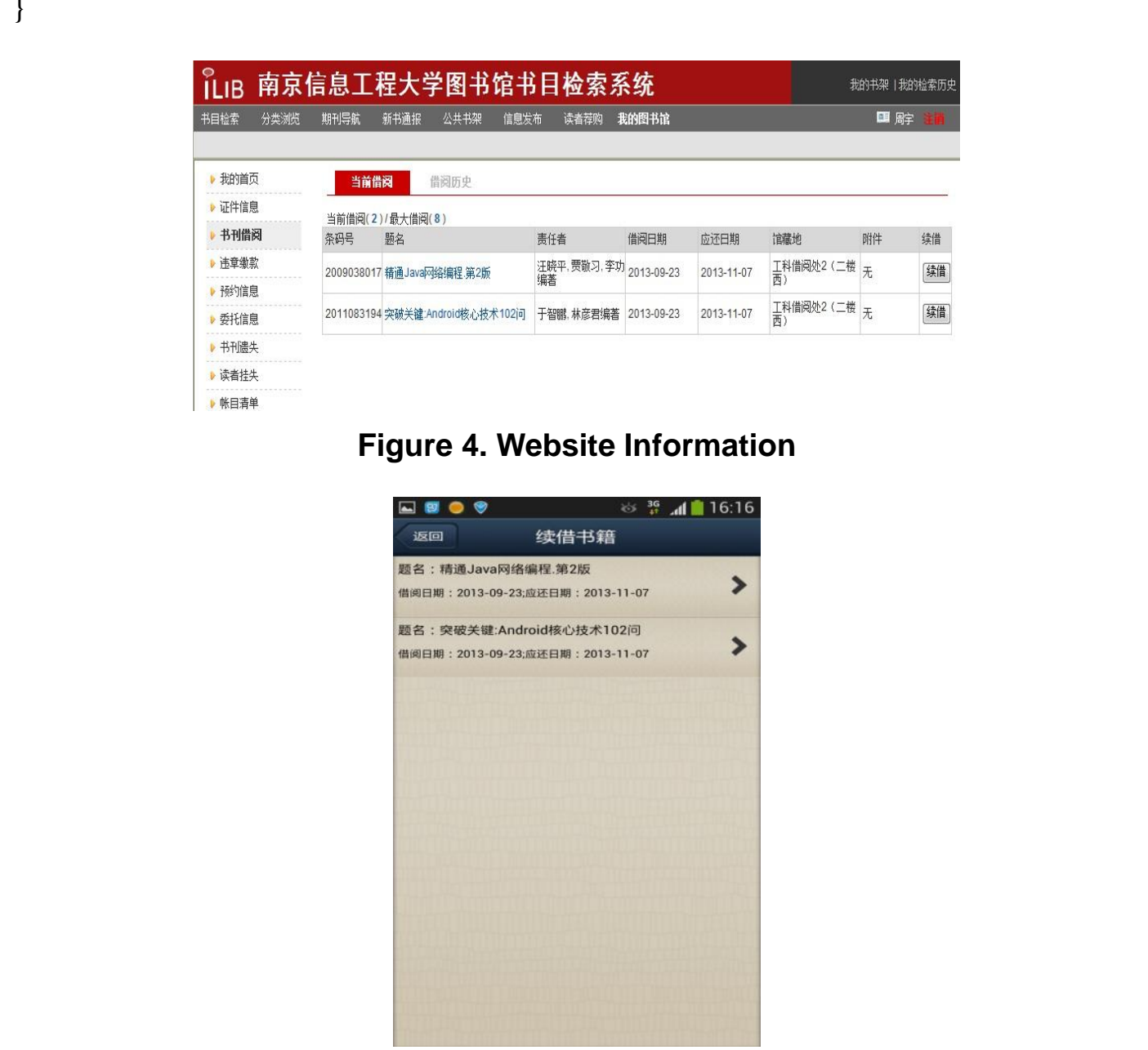

Figure 5. The Effect of Client Parsed 


\section{Resolution of Information Principles and Research}

By Crawling the pages, the program can get a page's HTML documents, but not all the content is needed, so in order to gain useful information, the system has to parse HTML document.

\subsection{Method}

WebView, regular expressions, SAX, HtmlParser and jsoup are commonly used when Android is parsing HTML files. WebView can use LoadUrl to display Web content and image files directly, but it can't parse out some part of the data flexibly. SAX is one way to parse XML documents. SAX can only parse XHTML files, and it parses slower than other ways. Besides, a file needs tens to hundreds of milliseconds to be parsed. The parsing efficiency of Regular expression is still low, but it can be combined with cut out or extract the needed content. HTMLParser is more flexible, but its references are fow, it's difficult to use them. In Android development, a good HTML parser should be introduced here --Jsoup parser. It can parse HTML by various ways, it can resolve a fRL address, and also parse a HTML text. Therefore, it is very easy to use. Considering the high efficiency and low cost, this paper's program applies Jsoup parser to extract useful information, and Figure 6 shows the class hierarchy of Jsoup parser.

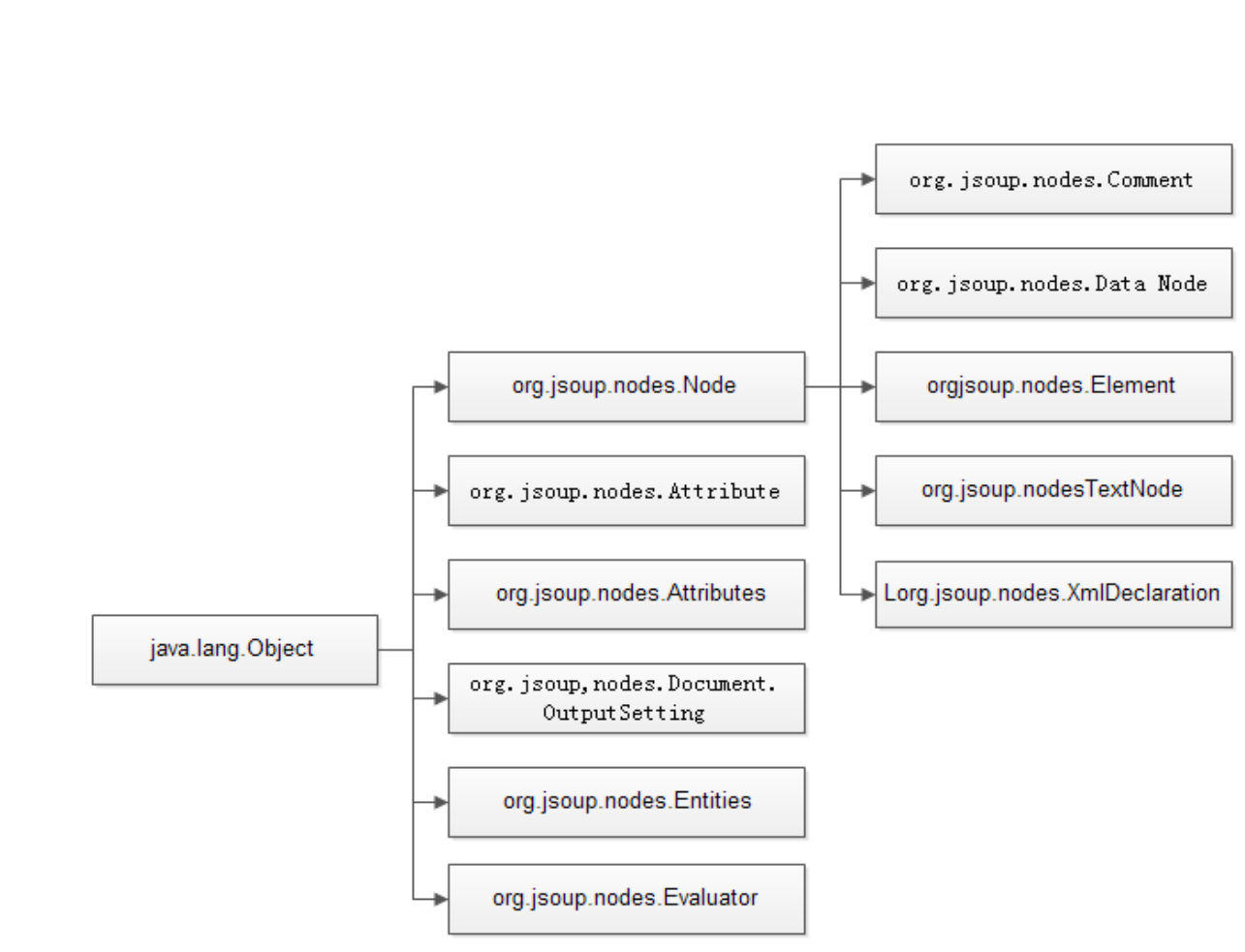

Figure 6. Class Hierarchies of Jsoup Parser

\subsection{Implementation of the Program}

Before parsing Jsoup, it is necessary to observe the structure of an HTML document, find the location of the required information, and make clever use of some syntax of Jsoup to resolve. Take resolving the "sky news" pages as an example. By observing the HTML document, the headline of the news can be found between <div id="title "> and $<$ / div $>$, the release time of the news and the author are between $<\operatorname{div}$ id = "ptime" $>$ and $</$ div $\rangle$, the specific content of the news is between $\langle\operatorname{div} \mathrm{id}=$ "content" $\rangle$ and $\langle/ \operatorname{div}\rangle$. By 
using the getElementById () of Jsoup, the information range can be decided, and then,parse the content and pictures depending on the specific situation. Figure 7 is the content of the news website and Figure 8 is the display of client, the key code is shown as follows:

Document doc $=$ Jsoup.parse $\left(\mathrm{Html} \_\right.$doc $)$;

$/ /$ title

xinxi1 = doc.getElementById("title").text();

//Source

Element content $=$ doc.getElementById("ptime");

xinxi2=Jsoup.parse(content.html().replace("\&nbsp;","\$")).text().replace("\$"," ");

$/ /$ the content of article

Element content2 =Jsoup.parse(doc.getElementById("content").html().replace("<br $/>$ ", $"</ \mathrm{p}\rangle\langle\mathrm{p}\rangle "))$;

Elements content $3=$ content 2 .select("p");

for $($ int $\mathrm{i}=0 ; \mathrm{i}<$ content 3 .size ()$; \mathrm{i}++)$

\{

Elements content $4=$ content $3 . g e t(i) \cdot \operatorname{select}(" \mathrm{img}[\mathrm{src}] ") ;$

if $($ content $4 . \operatorname{size}() !=0)$

\{

String wangzhi=content $4 . \operatorname{attr}(" s r c ")$;

Bitmap zy=getPicture(url_first+wangzhi);

list_href_1.add(zy);

list_href_3.add("1");

\}

else

\{

p_content $=$ content 3 .get $(\mathrm{i}) \cdot \operatorname{text}()$,

list_href_2.add(p_content);

list_href_3.add("2");

$$
\text { \} }
$$

\}

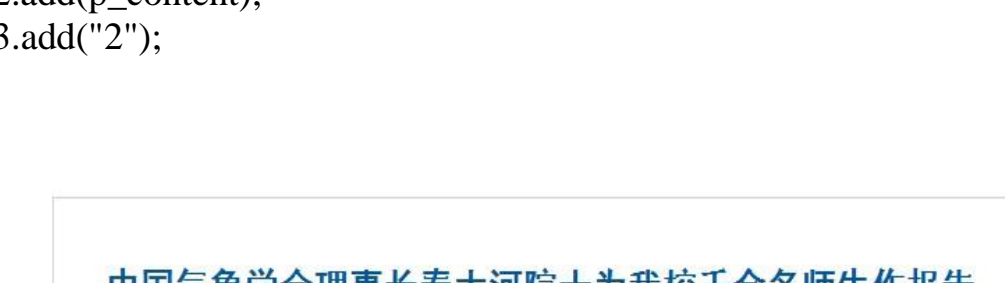

中国气象学会理事长秦大河院士为我校千余名师生作报告

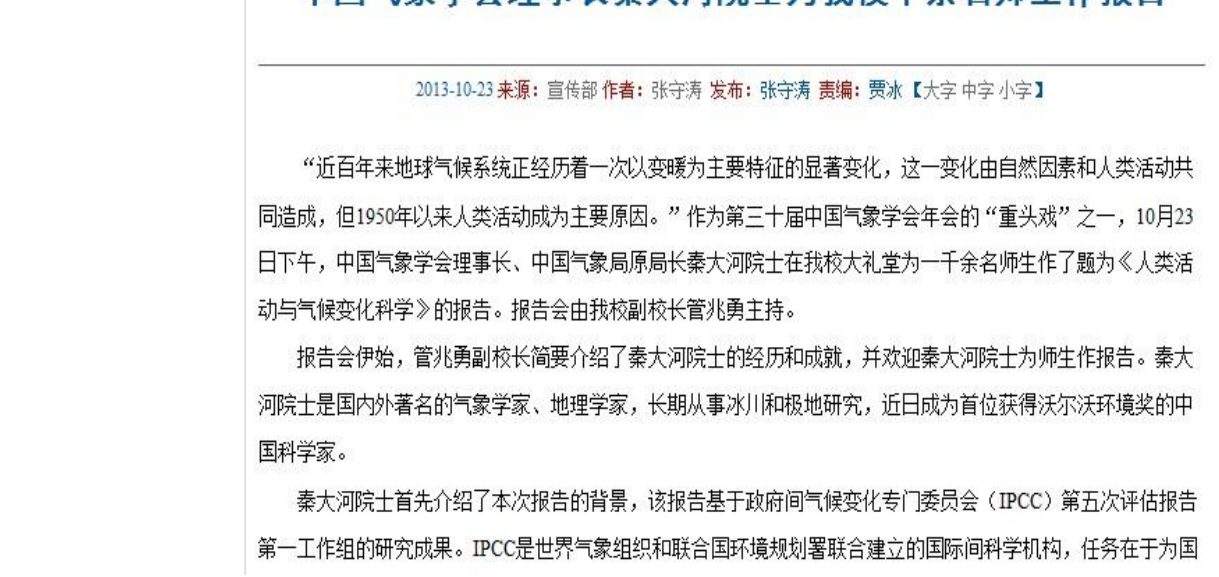

Figure 7. The Content of the News Website 


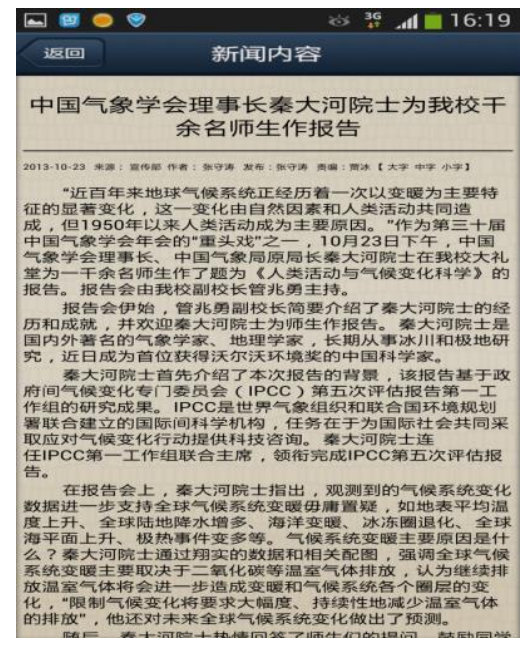

Figure 8. The Display of Client

\section{Achievement of Storing Information by SQlite Database}

Since most of the functions of this software have to be reakized through the network, and some information does not change frequently, such as.Dean's curriculum schedule and grades, basically change once in a semester, in order to save flow, the system uses Sqlite database to store information.

\subsection{Methods}

Sqlite is a free, source-open database that is irespective of the underlying database. It s upports furthest 2048GB of data and takes up less than 1MB of memory. It is easy to oper ate and has powerful database function. Considering these advantages, the Dean's data of this software applies the Sqlite database.

\subsection{Program Implementation}

The users have to input theirpersonal information to obtain the information from the Dean Website when the first time they login, and then the system will store the useful information parsed out by Jsoup into database. Figure 9 is the display of a certain day's curriculum byclient. The key code associated with Sqlite database operations is shown as follows:

mydb_jiaowuchu.delete(Table_Name2, null, null);

mydb_jiaowuchu.execSQL(Create_Table2); // Create a table

ContentVaruescr2=new ContentValues();

Document doc3;

doc3 $=$ Jsoup.parse $\left(\mathrm{Html} \_\mathrm{doc}\right)$;

String temp1 = doc3.html().replace("\&nbsp;", "空");

doe3 = Jsoup.parse(temp1);

Elements content5 = doc3. select("td[valign=top]");

for (Element con : content5) \{

cv2.put(Course, con.text().toString());

mydb_jiaowuchu.insert(Table_Name2, null, cv2);

\}

// Update the database

mydb_jiaowuchu.close(); 


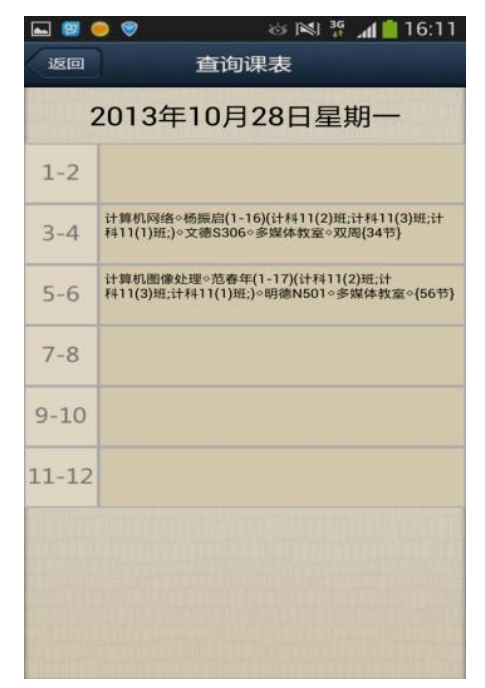

Figure 9. The Day's Curriculum

\section{System Testing}

This test involves three environments: hardware, software and networks. The hardware environment is Y460 Lenovo laptop and Samsung 19508 mobile phone. For the software, the laptop's operating system is windows 7 and the mobile phone's is Android 4.2.2. In addition, the network environment is $10 \mathrm{M}$ broadband China Mobile, WIFI LAN and GPRS network of China Mobile.

\subsection{Module of Registry Testing}

This module includes the functions of curriculum query and students' scores query, which is shown in Figure 10. And the login interface of Dean Website is shown in Figure 11 .

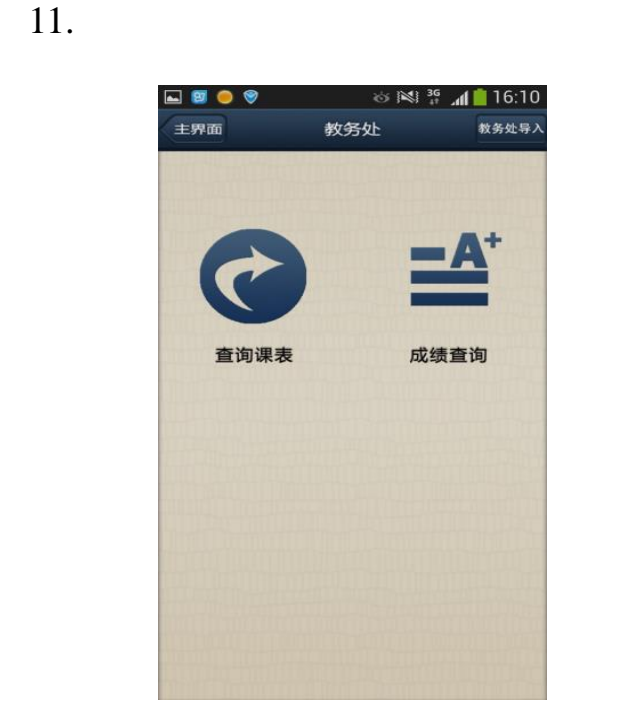

Figure 10. Main Interface of Dean Module

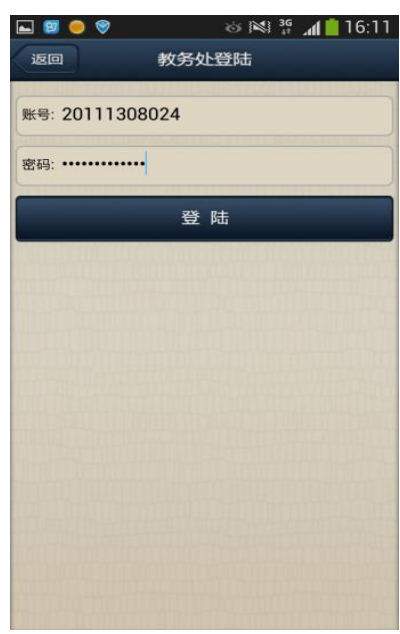

Figure 11. Dean Login

After extensive testing of this module, the curriculum query and students' scores query work very successfully. Test results are shown in Figure 12. 


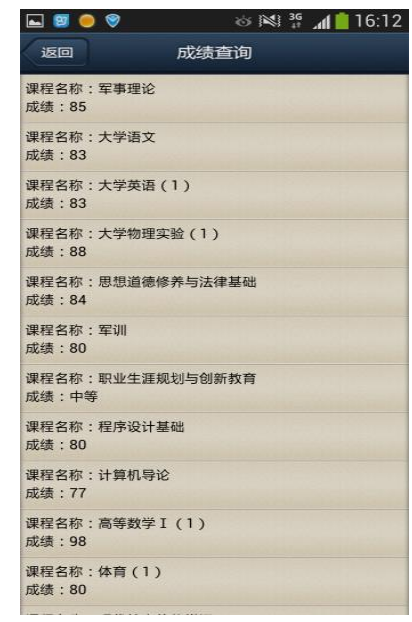

Figure 12. Scores Query

\subsection{Module of Library Testing}

This module includes functions of retrieving books and renewing books. The interface is shown in Figure 13.

After extensive testing of this module, the fun tion of retrieving books and renewing books work very successfully. Test results are shown from Figure 14 to Figure 18. Figure 14 is the interface of book retrieval, for which users can search books by entering the name of the book. Figure 15 is the interface of the results by searching books about Android on the button Next and Previous can be used to check all search results. Figure 16 is the login interface of library system and Figure 17 is the interface of checked out books, while the results of renewing books are shown in Figure 18.

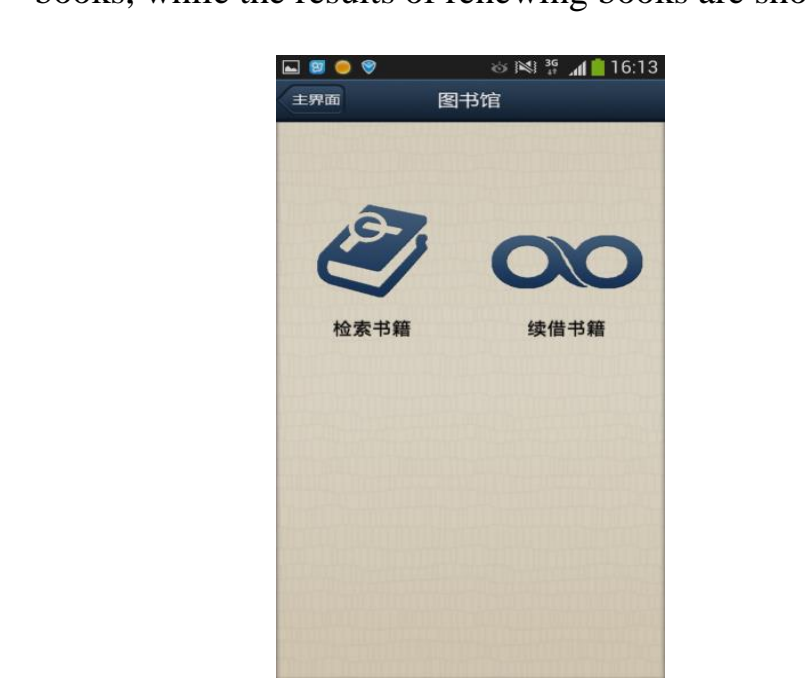

Figure 13. Module of Library

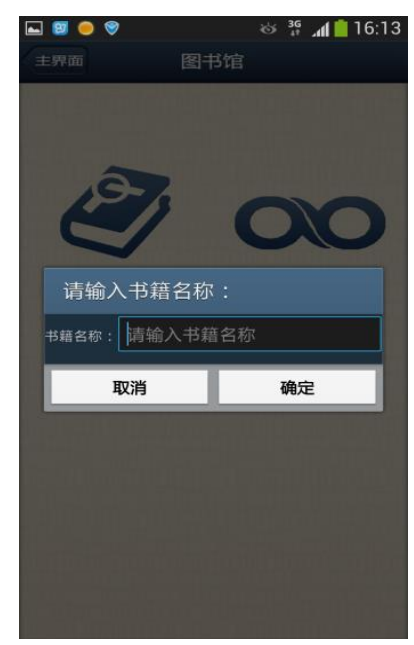

Figure 14. Book Retrieval 


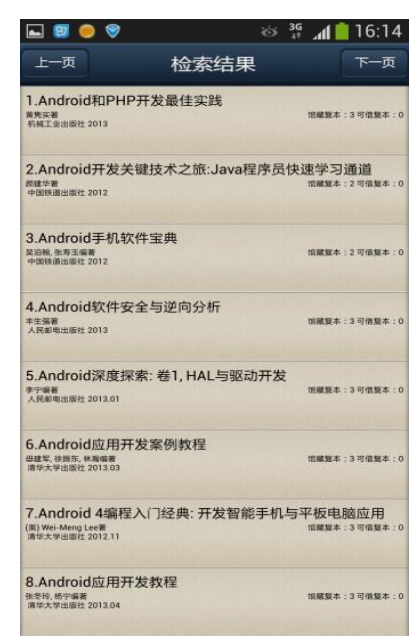

Figure 15. Results of Searching

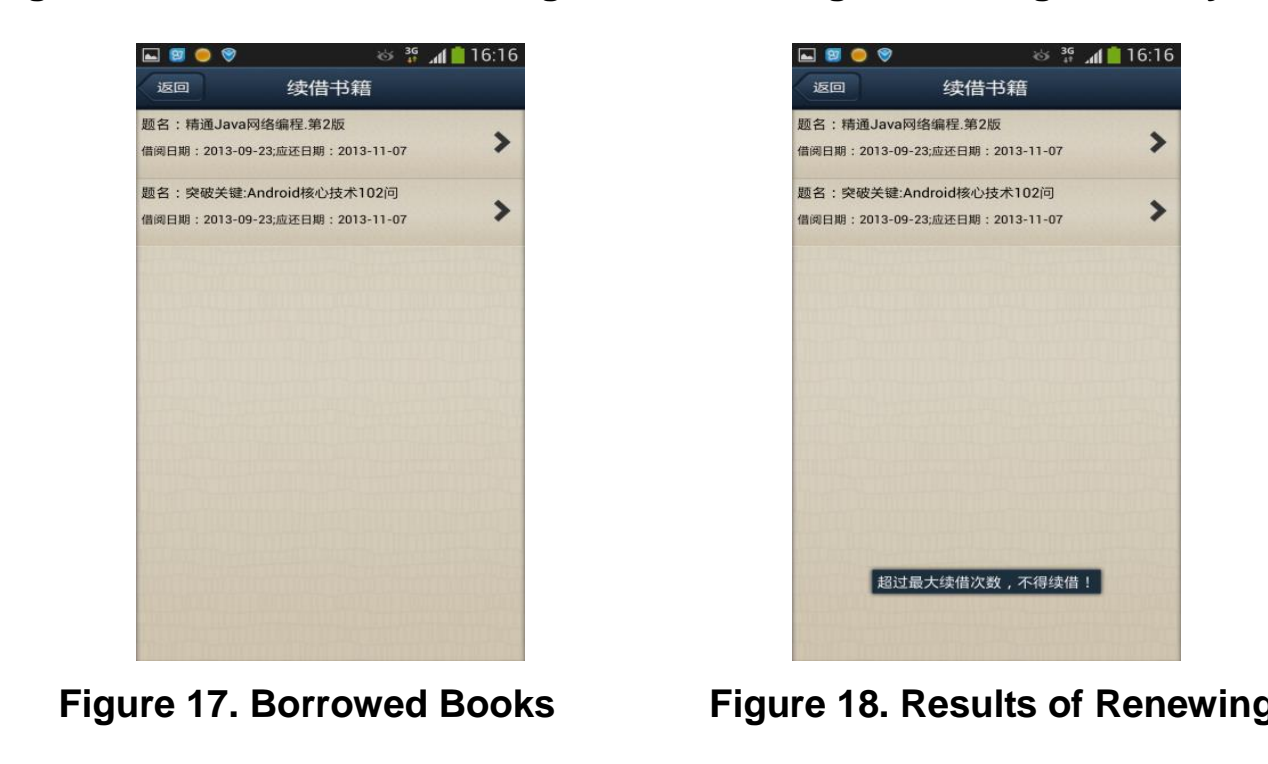

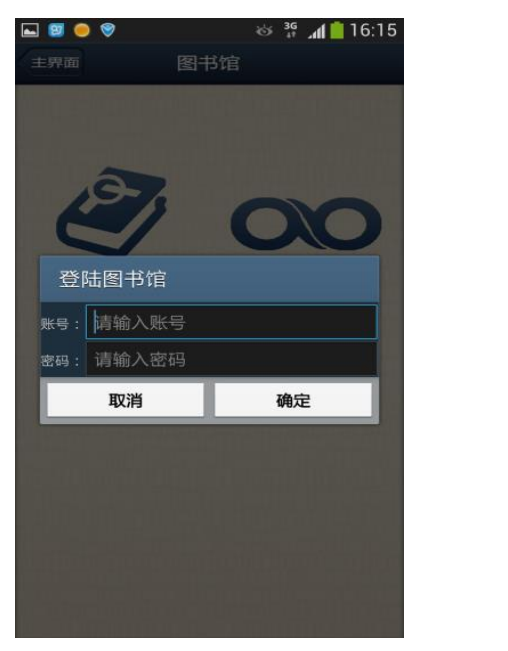

Figure 16. Login Library

Figure 18. Results of Renewing

\subsection{Module of Information Bulletin Testing}

This module contains the functions of viewing content of School Information Notice website in campus network and outside campus network. The function works very successfully after extensive testing of this module.

\section{Acknowledgments}

This aper is a revised and expanded version of a paper entitled "Semantic-element-bas ed Defining Approach for Model Transformation Rules" presented at CIA 2014, Angeles City (Clark), Philippines, April 24 -26, 2014. This research work was supported by the Na tural Science Foundation of China youth project (61103142). It was also supported by the by the Industrial Strategic Technology Development Program (10041740) funded by the Ministry of Trade, Industry and Energy (MOTIE) Korea.

\section{References}

[1] Information Technology Co., Ltd. Qingdao DongHe Android Programming [M] Beijing: Electronic Industry Press, (2012).

[2] D. Wang, "Android web development and detailed explains of applications", Beijing: The People's Posts and Telecommunications Press, (2012). 


\section{Authors}
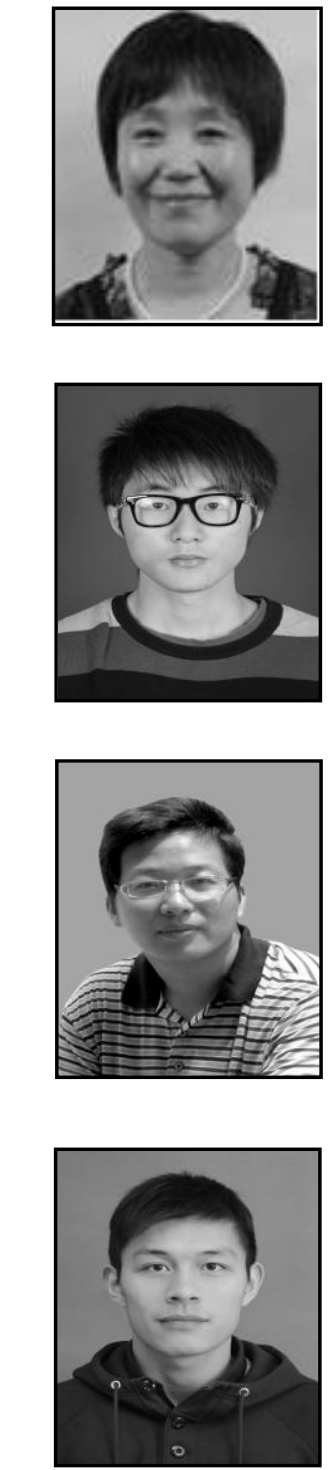

Shen Liu, he was-born on April, 29th in 1992. He is a third-grade student in Nanjing University of Information Science and Technology. His major is computer science and technology. He is keen on photo shop and image processing .Currently he is devoted to image processing and android application development.

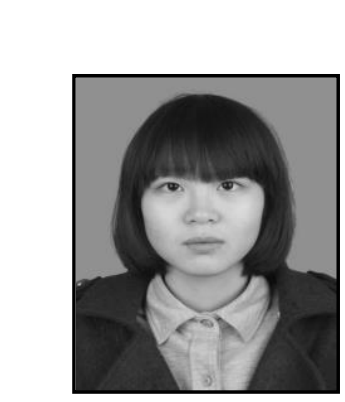

Jie Qiao, she was born on February, 16th in 1995. Currently she is a third-grade student in Nanjing University of Information Science and Technology. Her major is computer science and technology. Her areas of interest are fingerprint identification and android application development.

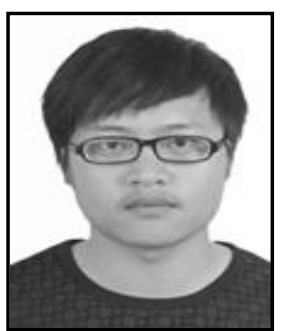

Zhenyu Han, he was born in October, 5th in 1991 and right now he is a senior in Nanjing University of Information science and technology. He will also receive his bachelor degree from University of North Carolina at Pembroke in 2014. His major is computer science and his research interest is programming and $3 \mathrm{D}$ implementation. 